NP8 (continued)

Objective: To describe Get Fruved year 03 components and outcomes.

Description: Get Fruved is a health promotion intervention utilizing Community Based Participatory Research to develop and test a social marketing and environmental change intervention to prevent unwanted weight gain among older adolescents. The intervention was developed, refined, and pilot/feasibility tested at four intervention colleges with four other colleges as control. One high school (HS) served as a site for initial development and feasibility testing of the program and data collection tools.

Evaluation: In 2015-2016 (college wave I), 578 nonfirst year students and 1,148 first year college students (FYCS) completed physical assessments and online surveys and 4,595 FYCS completed online-only surveys. In fall 2016, wave II online survey assessments were completed with 2,491 new FYCS and 237 HS students. Weight was the primary outcome, but since the intervention focused only on obesity-prevention, healthrelated behaviors, numerous health-related behaviors were assessed.

Conclusions and Implications: Two-year post assessments with college wave I, and one-year post assessments with college wave II will be completed in May 2017. As of year 03, twelve new instruments/methods have been developed/validated, three college courses created, seventy-five presentations conducted, and a college toolkit (providing a systematic approach to promoting health for obesity prevention) has been developed for national testing and dissemination. In year 04, intervention refinement and feasibility testing will occur on eight additional HS campuses. In years 04 and 05 the toolkit will be tested for effectiveness using a Randomized Control Trial design on new college and HS campuses.

Funding: 2014-67001-21851

\section{NP9 Using the Child as Change Agent for Preventing Childhood Obesity in Rural Georgia: Outcome Results on BMI for Age and Home Environment}

Marsha Davis, PhD, University of Georgia; Courtney Still,PhD, RD, courtms@uga.edu, 116E Rhodes Hall, Health Sciences Campus, Athens, Georgia 30602; Andrea Scarrow, MAL, University of Georgia;

Frances McCarty, PhD, George Washington University; Richard Christiana, PhD, Appalachian State University; Richard Lewis, PhD, University of Georgia; Rebecca Mullis, PhD, RD

Objective: This study aims to test effectiveness of a school-based curriculum, utilizing children as change agents, supplemented by family activities, to prevent childhood obesity.

Description: The Action Pack Families study is a group-randomized trial that follows children from the beginning of 3rd grade to the end of 5 th grade. These analyses consider changes in child body mass index for age from baseline (beginning of 3rd grade) to the end of 5th grade and opportunities for healthy eating and physical activity in the child's home environment.

Evaluation: Data were collected at the beginning of 3rd grade (baseline) and the end of 5 th grade in 10 schools. Child height and weight were collected at school by the research team; diet, physical activity, and home environment factors were self-reported by parents using a paper-pencil survey. We will present data from baseline to the end of 5th grade, focusing on child weight status.

Conclusions and Implications: Using children as change agents may be an effective way to alter the home environment and prevent childhood obesity.

Funding: 2012-68001-19625

\section{NP10 Engaging Youth as Advocates to Create Healthy Snacking Zones Around Rural Schools - Year 5}

Nancy Findholt, PhD, RN, findholt@ohsu.edu, Oregon Health \& Science University, One University Boulevard, La Grande, OR 97850; Betty Izumi, PhD, MPH, RD, Portland State University; Jackilen Shannon, PhD, MPH, $R D$, Oregon Health \& Science University; Thuan Nguyen, PhD, MD; Carole Smith, MEd, Oregon State University Extension Service

Objective: This quasi-experimental study evaluated an intervention that engaged 4-H youth participants in advocacy for "healthy snacking zones" within 5 elementary/ middle schools and nearby small food stores in a rural Oregon county.

Description: Youth engagement occurred through participation in 4-H "SNACZ clubs," which were established in each intervention school. Youth club members received nutrition lessons and, with support from adult club leaders, planned and implemented outreach projects to promote healthy snacking among their peers and encourage environmental change within their schools and the food stores.

Evaluation: Mixed methods were used to assess changes in school and store environments, as well as changes in children's snacking behaviors and BMI.

Conclusions and Implications: After the SNACZ program, intervention teachers had greater overall nutrition knowledge ( $\mathrm{p}=0.098)$ and were less likely to use food rewards for students, including sweetened drinks (0.053), than teachers in control schools. Mean availability of healthy snacks/beverages in food stores near intervention schools increased by $21 \%$, while mean availability of these products in stores near control schools decreased by $19 \%$. Students' mean consumption of unhealthy snacks/beverages outside of school decreased in both intervention and control sites, but the magnitude of the change was substantially greater in 